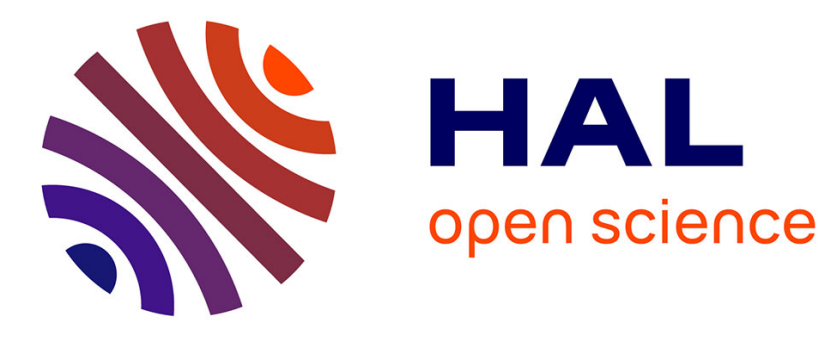

\title{
Drop Formation in Non-Newtonian Fluids
}

Mounir Aytouna, Jose Paredes, Noushine Shahidzadeh-Bonn, Sébastien

Moulinet, Christian Wagner, Yacine Amarouchene, Jens Eggers, Daniel Bonn

\section{To cite this version:}

Mounir Aytouna, Jose Paredes, Noushine Shahidzadeh-Bonn, Sébastien Moulinet, Christian Wagner, et al.. Drop Formation in Non-Newtonian Fluids. Physical Review Letters, 2013, 110 (3), pp.34501. 10.1103/PHYSREVLETT.110.034501 . hal-00793062

\section{HAL Id: hal-00793062 \\ https://hal.science/hal-00793062}

Submitted on 28 Feb 2018

HAL is a multi-disciplinary open access archive for the deposit and dissemination of scientific research documents, whether they are published or not. The documents may come from teaching and research institutions in France or abroad, or from public or private research centers.
L'archive ouverte pluridisciplinaire HAL, est destinée au dépôt et à la diffusion de documents scientifiques de niveau recherche, publiés ou non, émanant des établissements d'enseignement et de recherche français ou étrangers, des laboratoires publics ou privés.

\section{(ㄷ)(1) $\$$}

Distributed under a Creative Commons Attribution - NonCommerciall 4.0 International 


\title{
Drop Formation in Non-Newtonian Fluids
}

\author{
Mounir Aytouna, ${ }^{1}$ Jose Paredes, ${ }^{1}$ Noushine Shahidzadeh-Bonn, ${ }^{1}$ Sébastien Moulinet, ${ }^{2}$ Christian Wagner, ${ }^{3}$ \\ Yacine Amarouchene, ${ }^{4}$ Jens Eggers, ${ }^{5}$ and Daniel Bonn ${ }^{1,2}$ \\ ${ }^{1}$ Soft Matter Group, Van der Waals Zeeman Institute, IoP, Science Park 904, Amsterdam, Netherlands \\ ${ }^{2}$ Laboratoire de Physique Statistique, ENS, 24 rue Lhomond, 75005 Paris, France \\ ${ }^{3}$ Experimentalphysik, Universität des Saarlandes, Postfach 151150, 66041 Saarbrücken, Germany \\ ${ }^{4}$ LOMA, Université Bordeaux 1, 351 cours de la Libération, 33405 Talence, France \\ ${ }^{5}$ Department of Mathematics, University of Bristol, University Walk, Bristol BS8 1TW, United Kingdom
}

\begin{abstract}
We study the pinch off dynamics of droplets of yield stress and shear thinning fluids. To separate the two non Newtonian effects, we use a yield stress material for which the yield stress can be tuned without changing the shear thinning behavior, and a shear thinning system (without a yield stress) for which the shear thinning can be controlled over a large range, without introducing too much elasticity into the system. We find that the pinch off remains very similar to that of constant viscosity Newtonian liquids, and consequently thinning in shear flow does not imply a thinning in elongational flow.
\end{abstract}

PACS numbers: 47.50. d, 47.55.D , 83.50.Jf

The process of drop formation is observed frequently in everyday life, and its features are well understood for the case of Newtonian fluids [1]. However, since many fluids encountered in practice do not have a simple flow behavior, recently the case of non-Newtonian fluids has attracted much attention [2-9,9-14]. The main feature that has been considered so far is the case of solutions of flexible polymers, which are stretched in the elongational flow near the pinch point, leading to a spectacular rise of the elongational viscosity. As a result, the formation of long-lived fluid filaments provides a striking visual difference between Newtonian and non-Newtonian breakup $[2,15,16]$.

However, shear-thinning [17] and yield stress [18] behaviors are at least as frequent features of nonNewtonian flow as elasticity. How does the thinning in shear flow affect the breakup dynamics, keeping in mind that the shear rate encountered during breakup is small in comparison to the rate of elongation [19]? In recent years, it has become standard practice in the literature on drop breakup to postulate the same rheological response for the extensional flow near breakup $[5,6,12]$ as for the thinning behavior in shear flow. Other studies have included both a yield stress and shear thinning, based on the same idea [7,8]. The main conclusion of the theoretical analyses of the breakup of shear-thinning fluids has therefore been that breakup proceeds faster than in the Newtonian case, increasingly so as the point of breakup is approached, since the elongation rate strongly increases close to breakup.

In this Letter, we investigate the breakup dynamics for fluids with well-defined (and nonelastic) non-Newtonian effects, which allow us to focus either on the shear thinning or the yield stress behavior. We find that contrary to previous theoretical analyses, breakup is described completely by the equations for the breakup of simple
Newtonian fluids, controlled by either a viscous-capillary or an inertial-capillary balance.

To study the dynamics of drop formation of the complex fluids in air, we use a high-speed video camera (Phantom V7 at 12000 frames/s). The controlled release of the drops is achieved using a syringe pump to set a low drop emission rate and a syringe with a needle of diameter $d=1.95 \mathrm{~mm}$. The rheological measurements for the same fluids, presented here were done by using a cone-plate geometry on a Physica MCR 300 rheometer.

As a yield stress system with variable yield stress, we make emulsions of castor oil droplets dispersed in water stabilized by $1 \mathrm{wt} \%$ sodium dodecyl sulfate; water is the continuous phase and the sodium dodecyl sulfate and the castor oil were purchased from Sigma. The drop size distribution in the emulsion was measured using confocal microscopy; the average drop diameter is $3.14 \mu \mathrm{m}$, with a size polydispersity of $19 \%$. Because of the small size of the droplets compared to the minimum radius of the neck, we do not anticipate any inhomogeneity during the thinning; indeed in the experiments the filament radius remains smooth. For the rheology of the emulsion, Fig. 1 shows that the classical Herschel-Bulkley model $\tau=\tau_{y}+k \dot{\gamma}^{n}$ provides a good description of the data. Varying the volume fraction, we can make the yield stress $\tau_{y}$ vary over several orders of magnitude, while the power-law exponent $n$ of the shear thinning remains remarkably similar and is always around 0.5 .

The evolution of the minimum neck radius (cf. Fig. 2) was obtained from sequences of rapid camera images; each experiment was repeated at least 5 times. The thinning of the neck is usually fitted to a power-law dependence; here we do the same and fit

$$
R_{\min }=A\left|t_{0}-t\right|^{\alpha},
$$




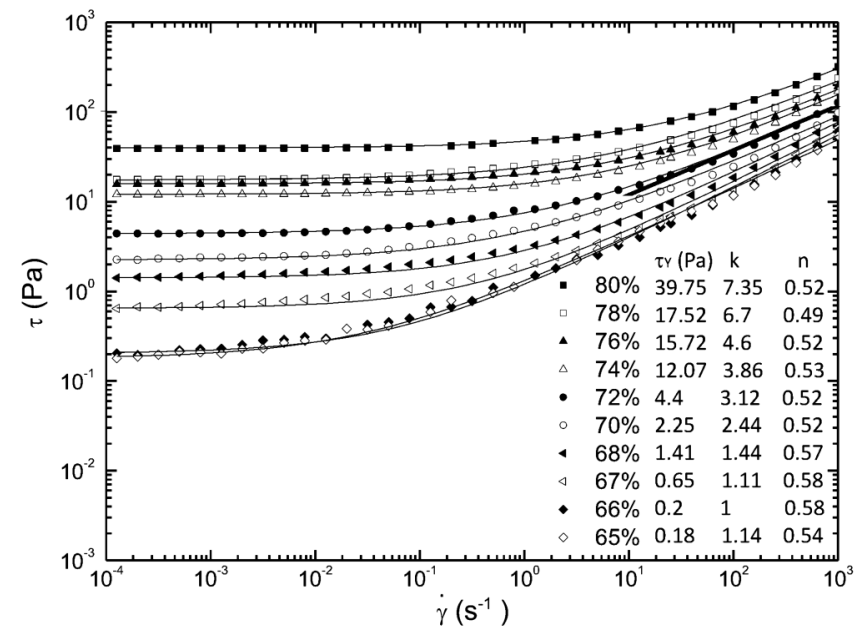

FIG. 1. Yield stress system: shear stress vs shear rate for several volume fractions of castor oil; the shear thinning expo nent is nearly constant, $n \approx 0.52 \pm 0.04$ (a line of slope $\frac{1}{2}$ has been added at the right hand side).

where $t_{0}$ is the breakup time and $\alpha$ is the power-law exponent. For Newtonian fluids, $\alpha$ is either $\frac{2}{3}$ or 1 , depending on which forces are balanced. If the elongational viscosity is rate dependent, the expectation is that $\alpha$ equals the power-law exponent of the thinning: $\alpha=n[5,7,8,12]$. However, in our filament thinning experiments (cf. Fig. 2), we find $\alpha \approx 0.67$ for low yield stress emulsions, and unity for very elastic emulsions (Fig. 3). Here the somewhat gradual transition is the result of fitting over a limited
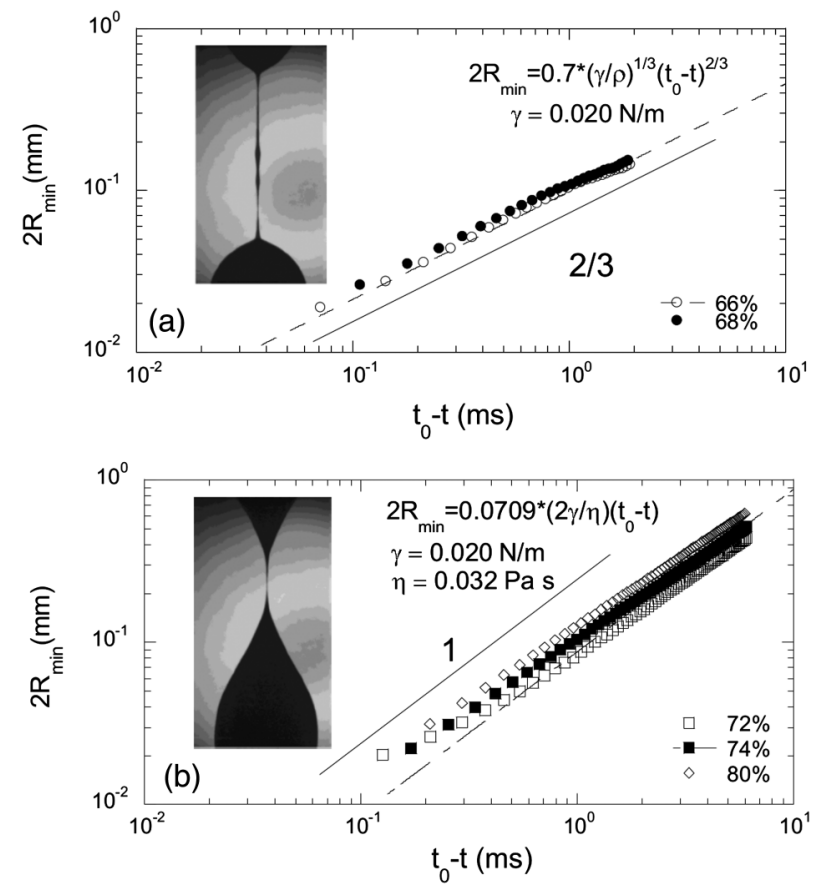

FIG. 2. The minimum neck diameter $R_{\min }$ of the emulsion vs time, with low and high concentrations of castor oil (powers $0.67 \pm 0.02$ for $\phi<70 \%$ and $0.99 \pm 0.03$ for $\phi>72 \%$ ). scaling range. This suggests that the emulsion behaves very similarly to Newtonian fluids, where for low viscosities one finds an exponent of $\frac{2}{3}$ (inertia-capillary regime), while for high viscosities a power of unity is observed (capillary-inertial-viscous or capillary-viscous regime) [1]. This picture is confirmed by the fact that at the crossover, a typical Reynolds number Re is of order unity, as shown in the inset of Fig. 3.

In addition, the main visual difference between viscous and inertial regimes is that for the former the breakup exhibits up-down symmetry around the breakup point, whereas the symmetry is broken if inertia is important. Indeed, the asymmetry coefficient defined in [20], which is plotted in Fig. 3, demonstrates a transition from asymmetric to symmetric, as the power-law exponent changes from $\frac{2}{3}$ to 1 .

Thus, the yield stress appears to be irrelevant and the breakup exponent is unrelated to the rheology altogether. To assess what the effective viscosity for the thinning is in the experiment, we can use the fact that for Newtonian fluids the rate of thinning in the viscous regime is given by the capillary speed $\gamma / \eta$, where $\gamma$ is the surface tension. However, determining the latter is in fact a difficult problem, since most if not all of the usual methods for determining surface tensions of liquids fail when the material has a yield stress.

We therefore developed a new method that makes use of the breakup images. Once the drop is formed, the conical shape of the interface (see the pictures in Fig. 4, inset) relaxes under the influence of the surface tension. This process stops as soon at the capillary pressure can no

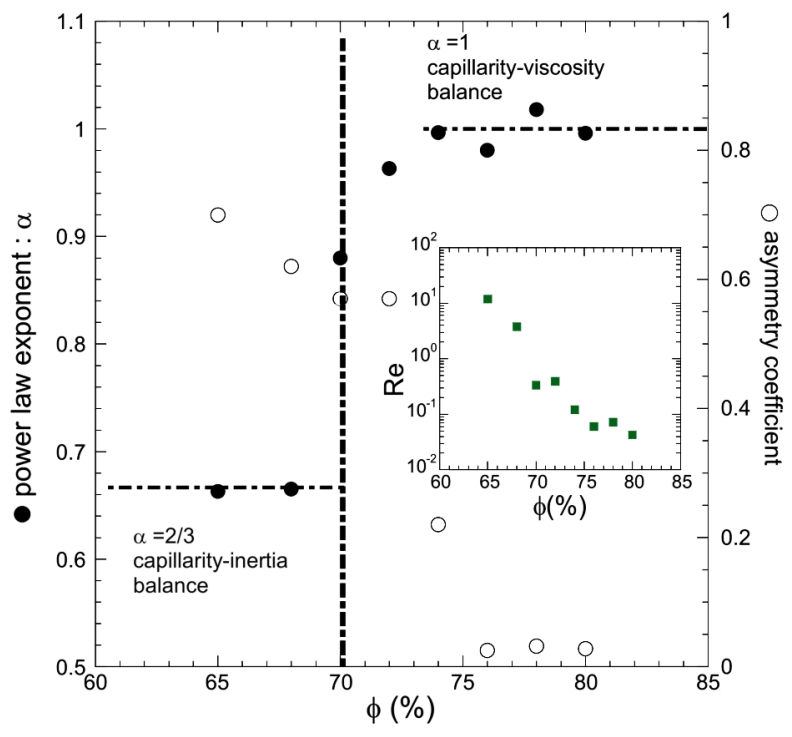

FIG. 3 (color online). Left scale: power law exponent from fit to the minimum neck radius (cf. Fig. 2) of the yield stress fluid vs volume fraction (black circle). Right scale: asymmetry coef ficient (open circles) In the inset, Reynolds number vs volume fraction. 


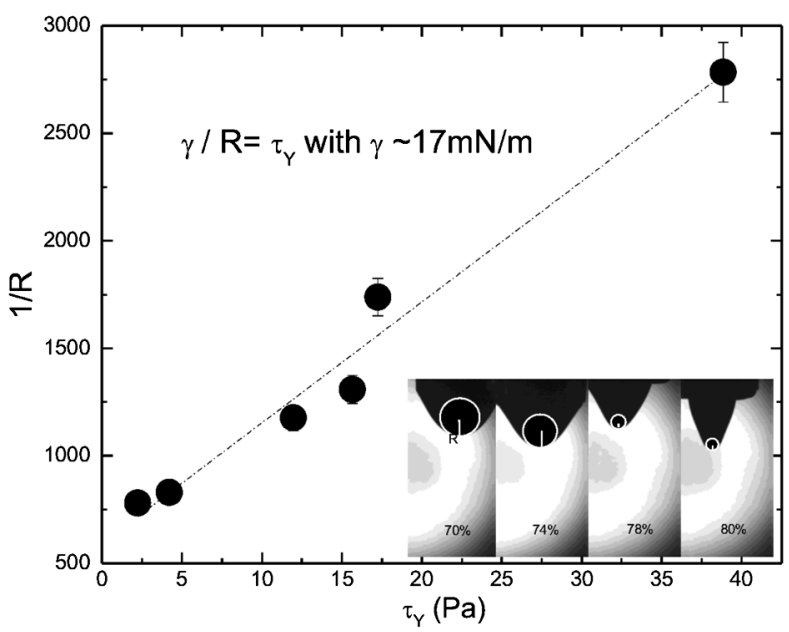

FIG. 4. Inverse radius of curvature vs yield stress for different volume fractions of castor oil. The inset shows the rounding of the interface after drop detachment.

longer overcome the yield stress of the material and so from measuring the radius of curvature, as indicated in the figure, the surface tension should follow from the force balance $\gamma / R=\tau_{y}$. We thus measure the curvature of the interface a long time after breakup when it no longer moves, and plot the curvature as a function of the yield stress. We find roughly a straight line, the slope of which should be the surface tension, as seen in Fig. 4.

We find a surface tension $\gamma=17 \pm 4 \mathrm{mN} / \mathrm{m}$, close to the expected value, since both the oil phase and the surfactant rich water phase have a surface tension $\gamma \approx$ $20 \mathrm{mN} / \mathrm{m}$. Then, knowing the surface tension, we can obtain the effective viscosity from the filament thinning rate in the viscous regime. For instance from the data shown in Fig. 2, we conclude that the capillary velocity is on the order of $0.025 \mathrm{~m} / \mathrm{s}$ for the $74 \%$ sample, corresponding to a viscosity of $0.032 \mathrm{~Pa}$ s. Comparing to the rheology, this suggests that the value of the viscosity deduced from the breakup experiment corresponds to a typical shear viscosity at high shear rates.

Therefore, to study the relationship between pinch-off and shear-thinning rheology in more detail and more quantitatively, we will consider a simpler system. We use aqueous xanthan solutions and solutions of xanthan in glycerol-water mixtures (the glycerol was obtained from Sigma) to be able to control the shear thinning. We use xanthan since these solutions are known to be very strongly shear thinning, and the shear thinning exponent can be changed by changing the concentration, expressed in ppm (weight parts per million). In addition, the xanthan introduces very little elasticity into the system, which is necessary to separate elastic from shear thinning effects [21].

The data for the thinning of the neck is shown in Fig. 5, which is again fitted by (1), the scaling exponent being reported in Fig. 6. We find that the power remains constant

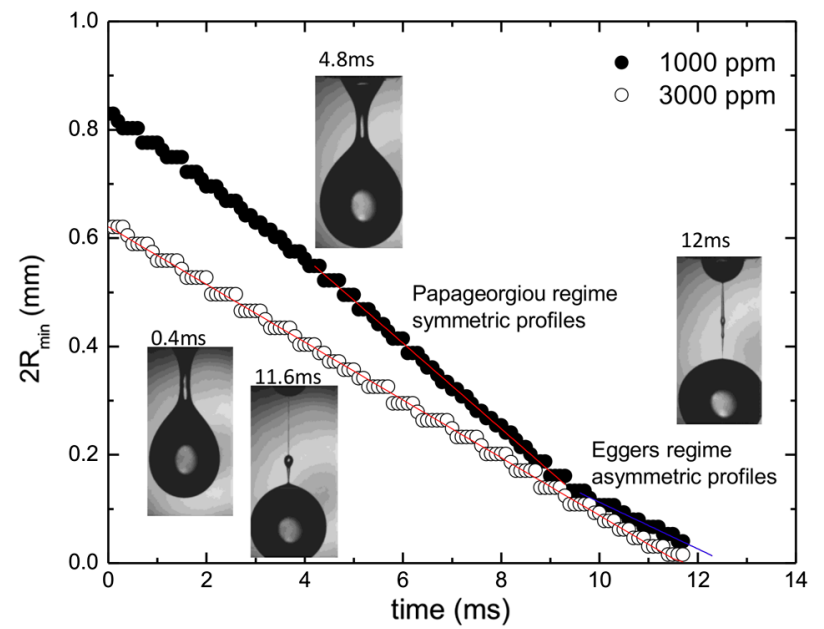

FIG. 5 (color online). Evolution of the diameter $2 R_{\min }$ for low and high concentrations of xanthan in water $[1000 \mathrm{ppm}$ (slope $d R_{\min } / d t=0.0395 \mathrm{~m} / \mathrm{s} ; \eta=0.128 \mathrm{Pas}$ ) and $3000 \mathrm{ppm}$ $\left.\left(d R_{\min } / d t=0.0265 \mathrm{~m} / \mathrm{s} ; \quad \eta=0.192 \mathrm{Pas}\right)\right]$. Error bars are smaller than the symbols.

around 1, and does so both for aqueous xanthan solutions and for xanthan in glycerol-water mixtures; again, each experiment was done at least 5 times to ensure the reproducibility of the results. The check with glycerol was done to make sure inertia could not influence the results. From the data, we show that again the rheology does not influence the breakup dynamics, although the shear-thinning exponent varies over a large range.

This allows us to compare to a constitutive equation for xanthan, which behaves like a suspension of rigid rods [21]. The measured elongational viscosity, found from the slope of $R_{\min }$ in the viscous-capillary regime (taking $\eta_{e}=3 \eta=0.213 \gamma /\left|\dot{R}_{\min }\right|$ [1]), is reported in Fig. 7. A rigid rod will align in the direction of the extensional flow, increasing the viscosity, since the elongational stress is supported by the tension in the rods. Aligned rods are in a stable equilibrium position, so even moderate rates of

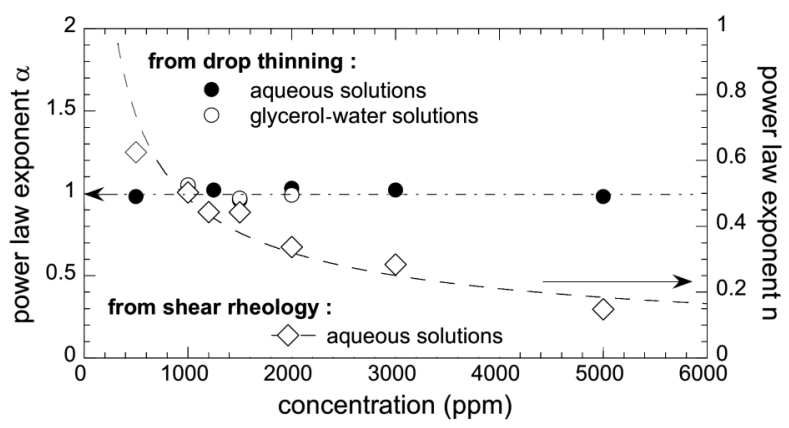

FIG. 6. Left scale: power law exponent $\alpha$ from fit to $R_{\min }$ (cf. Fig. 5), vs concentration of xanthan in water as well as in glycerol water mixtures. Right scale: power law exponent $n$ from the power law variation of the viscosity (Fig. 8) for aqueous xanthan solutions. 


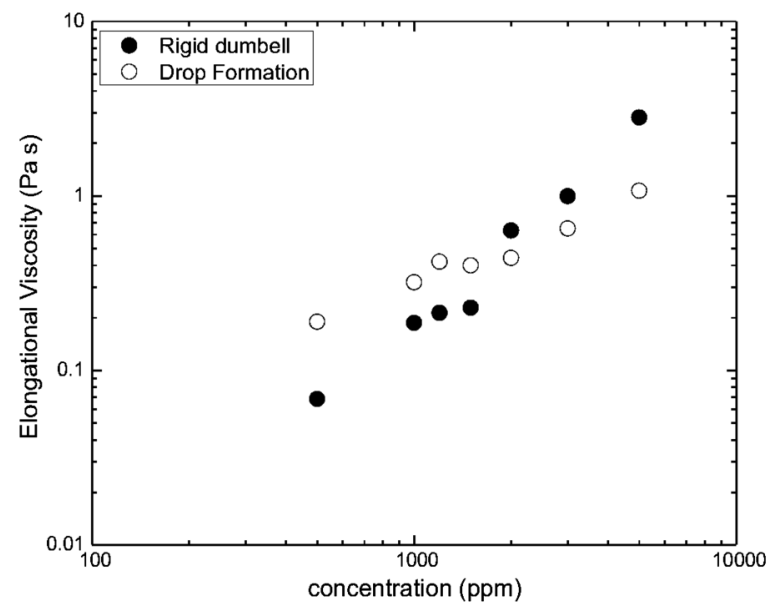

FIG. 7. Elongational viscosity calculated from $\left|\dot{R}_{\text {min }}\right|$ (solid circle) and from (2) (open circle) vs xanthan concentration.

extension will lead to almost complete alignment, and the elongational viscosity reaches the constant value [21]:

$$
\eta_{\mathrm{el}}=3 \eta_{s}+6 N k_{\mathrm{B}} T \lambda .
$$

Here $\eta_{s}$ is the solvent viscosity $\left(10^{-3} \mathrm{Pas}\right), N$ the number density of the polymers in the suspension, $T$ the temperature, and $\lambda$ the relaxation time of the polymer solutions, which we determine from shear measurements. As seen in Fig. 7, measured and theoretical values for the extensional viscosity are in reasonable agreement, so we have obtained a quantitative picture of the relation between shear and elongation viscosity in a strongly shear thinning system.

The predicted shear viscosity of the same rigid dumbbell model, on the other hand, shows a behavior that is opposite to the extensional behavior in every aspect. Increasing alignment in the shear direction leads to a decreasing viscosity with shear rate, as particles become better oriented in the direction of shear, offering less and less resistance to the flow. However, the aligned state is unstable in

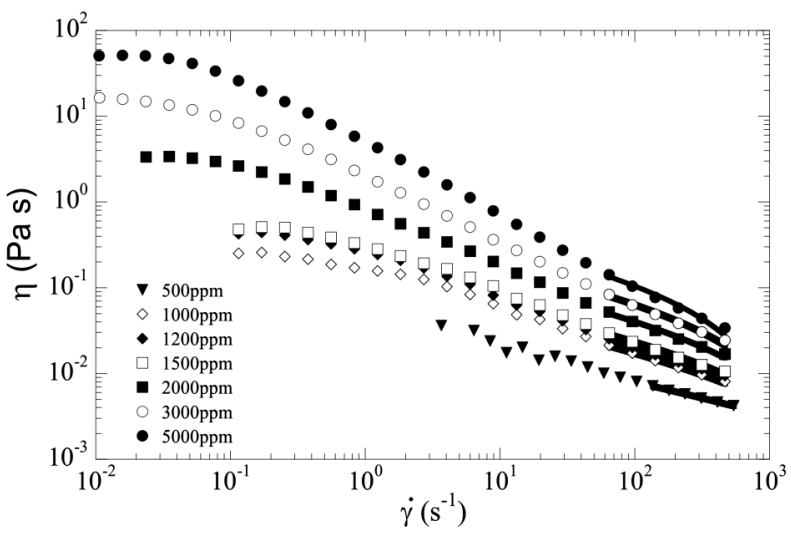

FIG. 8. Viscosity vs shear rate, for several concentrations of aqueous xanthan; straight lines are fits of (3) to the high shear end. shear flow, so the rod flips continuously between two equilibria, the transient time decreasing gradually with $\dot{\gamma}$, leading to the power-law dependence [21]:

$$
\eta(\dot{\gamma})=\eta_{s}+0.678 N k_{\mathrm{B}} T \lambda^{2 / 3} \dot{\gamma}^{-1 / 3} \text {. }
$$

In Fig. 8 we fit (3) to the high-shear end of our shear measurements, treating $\lambda$ as an adjustable parameter, used in (2) to calculate the shear viscosity.

In conclusion, we have studied the breakup of nonNewtonian fluids for the cases of shear thinning and yield stress materials; we conclude that both the emulsion and the polymer solution behave very similarly to Newtonian fluids: neither the yield stress nor the shear thinning come into play. Both the shear and the elongational viscosity are modeled quite well by the rigid dumbbell model for high shear rates. During drop formation, the elongational viscosity is always saturated at its high deformation rate value.

Our physical picture does not take into account the possible formation of complex structures created by highly interacting particles. Measurements of a liquid crystalline fluid in the smectic phase have found an extensional rheology in line with shear measurements [10]. However, other liquid crystalline phases showed a strong discrepancy between shear rheology and breakup measurements [11]. A correspondence between shear and elongation rheology was also reported in the case of even more complex (and less well characterized) fluids like ketchup, shaving foam, and hair gel $[22,23]$. We have to conclude that there may be liquids forming complex networks, whose rheology is beyond our current understanding.

[1] J. Eggers and E. Villermaux, Rep. Prog. Phys. 71, 036601 (2008).

[2] Y. Amarouchene, D. Bonn, J. Meunier, and H. Kellay, Phys. Rev. Lett. 86, 3558 (2001).

[3] C. Wagner, Y. Amarouchene, D. Bonn, and J. Eggers, Phys. Rev. Lett. 95, 164504 (2005).

[4] C. Clasen, J. Eggers, M. A. Fontelos, J. Li, and G. H. McKinley, J. Fluid Mech. 556, 283 (2006).

[5] M. Renardy and Y. Renardy, J. Non Newtonian Fluid Mech. 122, 303 (2004).

[6] P. Doshi and O. A. Basaran, Phys. Fluids 16, 585 (2004).

[7] N. J. Balmforth, N. Dubash, and A.C. Slim, J. Non Newtonian Fluid Mech. 165, 1139 (2010).

[8] N. J. Balmforth, N. Dubash, and A.C. Slim, J. Non Newtonian Fluid Mech. 165, 1147 (2010).

[9] D. Porter, J. R. Savage, I. Cohen, P. Spicer, and M. Caggioni, Phys. Rev. E 85, 041701 (2012).

[10] J. R. Savage, M. Caggioni, P. T. Spicer, and I. Cohen, Soft Matter 6, 892 (2010).

[11] A. A. Verhoeff and H. N. W. Lekkerkerker, New J. Phys. 14, 023010 (2012).

[12] O. E. Yildrim and O. A. Basaran, J. Non Newtonian Fluid Mech. 136, 17 (2006). 
[13] O. E. Yildirim and O. A. Basaran, Chem. Eng. Sci. 56, 211 (2001).

[14] P. Doshi, R. Suryo, O. E. Yildirim, G. H. McKinley, and O. A. Basaran, J. Non Newtonian Fluid Mech. 113, 1 (2003).

[15] R. Sattler, C. Wagner, and J. Eggers, Phys. Rev. Lett. 100, 164502 (2008).

[16] R. Sattler, S. Gier, J. Eggers, and C. Wagner, Phys. Fluids 24, 023101 (2012).

[17] R. B. Bird, R. C. Armstrong, and O. Hassager, Dynamics of Polymeric Liquids (Wiley, New York, 1987), Vols. 1 and 2.
[18] P. Moller, A. Fall, V. Chikkadi, D. Derks, and D. Bonn, Phil. Trans. R. Soc. A 367, 5139 (2009).

[19] J. Eggers and T.F. Dupont, J. Fluid Mech. 262, 205 (1994).

[20] A. Rothert, R. Richter, and I. Rehberg, Phys. Rev. Lett. 87, 084501 (2001).

[21] D. Bonn and J. Meunier, Phys. Rev. Lett. 79, 2662 (1997).

[22] P. Coussot and F. Gaulard, Phys. Rev. E 72, 031409 (2005).

[23] F. M. Huisman, S. R. Friedman, and P. Taborek, Soft Matter 8, 6767 (2012). 